\title{
Simon sur les planches des Feluettes de Michel Marc Bouchard
}

\section{Entretien accordé à Marie Pascal, Université Dalhousie}

Comme Michel Marc Bouchard, Stéphane Lestage a étudié au département de théâtre de l'Université d'Ottawa. Après ces débuts dans le Britanicus de Racine au Centre National des Arts d'Ottawa, il est recruté par Brigitte Haentjens au Théâtre du Nouvel-Ontario de Sudbury pour y jouer le rôle de «Ti-Jean », personnage légendaire de la tradition orale du nord de l'Ontario. La saison suivante, il jouera dans Nickel, aussi du TNO, et c'est à cette occasion qu'il fera une tournée (Ontario, Québec et dans les maritimes) en compagnie de Michel Marc Bouchard. Suite à cette tournée, le dramaturge le recrute pour jouer Simon lors de la création des Feluettes à L'Atelier du CNA (Ottawa). De retour à Montréal, Stéphane jouera surtout à la télévision pendant quelques années, puis fera ensuite une transition vers les domaines plus techniques du cinéma et de la télévision.

M.P. Tu as joué Simon dans la lecture du Feluette de Michel Marc Bouchard en 1984. Je suis intéressée par ce personnage dont j'étudie la marginalité dans mon manuscrit L'écho des lisières et des bas-fonds - Rapports de force dans la transcréation québécoise. J'aimerais avoir ton avis personnel, en tant que comédien qui a donné chair au personnage la première fois, sur le premier visage de Simon et, accessoirement, sur les techniques de création de ce dramaturge que tu connais bien.

\section{Sur M.M. Bouchard :}

\section{M.P. En 1984, quand il t'a approché pour jouer Simon, que connaissais-tu du dramaturge ?}

S.L. En fait, si on remonte au temps où j'étais au CEGEP, j'avais déjà entendu parler de Bouchard au cours de ce qui s'appelait le «printemps des CEGEP». Pendant une fin de semaine, tous les CEGEP se regroupaient et une pièce de Michel Marc Bouchard avait été présentée. Je me souvenais de son nom. Puis, on est allé à la même université, même s'il était quelques années avant moi. Par la suite, j'ai eu une offre pour travailler au Théâtre du Nouvel Ontario et Michel Marc est venu se greffer à une des pièces qu'on mettait en scène là-bas, Nickel, portant sur la syndicalisation des mines dans les années 1930. On s'est donc rencontré en tant qu'acteurs, on a joué dans la même pièce, on a fait la tournée ensemble pendant quasiment deux ans. Quand la tournée s'est terminée, moi, de mon côté, j'avais prévu de retourner à Montréal et il m'a parlé de ce qu'il était en train d'écrire. J'ai accepté de participer aux lectures parce qu'on s'était bien entendu pendant la tournée... Michel, c'est un passionné. Puis c'était déjà un personnage assez connu dans le milieu théâtral franco-ontarien quand il est venu jouer chez nous avec Nickel, à Sudbury. C'était déjà un auteur prolifique, passionné, qui était acteur pour gagner sa vie.

\section{M.P. Comment se passent les répétitions ?}

S.L. Les feluettes, c'était un projet du Centre National des Arts à Ottawa qui proposait un espace, qu'on appelait «Le Studio », une espèce de laboratoire théâtral si on veut. Son projet avait été sélectionné par ce laboratoire et, avec plusieurs collègues de Nickel, on s'est regroupé dans cette salle. Michel avait déjà une bonne idée de la pièce qu'il voulait écrire mais il voulait travailler avec des acteurs pour incarner le texte plutôt que l'écrire reclus chez lui. Il y avait un processus de création là-dedans : chaque jour, il arrivait avec quelques scènes qu'il voulait travailler. S'il y avait du texte, on prenait le texte, on en rajoutait, on essayait d'improviser, de discuter des interactions entre les personnages pour les bâtir de 
manière plus cohérente. Un processus de cinq semaines dans le but de donner trois soirs de lectures où on présentait la pièce. Je me demande même si, entre les soirs, on ne faisait pas de modifications. Il travaillait énormément ses textes.

M.P. Quel type de dramaturge est M.M. Bouchard, si c'est possible de répondre à cette question, car j'imagine qu'il a dû évoluer entre ses pièces ?

S.L. Moi, je trouve que c'est un dramaturge poétique, qui réfléchit à la fois à des sujets universels mais qui sont souvent incarnés dans sa région de Roberval. Beaucoup de ses pièces se situent entre l'épopée et le huis-clos. C'est peut-être un peu contradictoire mais c'est quelqu'un qui est en mesure de faire d'un huis-clos une épopée. Je pense que c'est cet aspect qui enjoue les gens mais aussi la poésie de ses textes et le fait que les mots prononcés sont souvent des révélations. Son style me fait un peu penser à Marcel Dubé chez qui les personnages ont vraiment une leçon à nous donner. Il y a comme un trop-plein qui revient au tempérament québécois : ce besoin de garder les choses à l'intérieur pis quand ça sort, tout est arraché. La débâcle. Dans le cas des Feluettes, c'est une pièce qui a beaucoup évolué de notre lecture à l'adaptation cinématographique. La structure narrative n'est pas la même. Dans le film, la débâcle finale, c'est catastrophique.

\section{Sur Les feluettes :}

M.P. Tu parlais des trois lectures que vous aviez faites. Te souviens-tu de l'accueil et de la réception de la pièce à ce moment-là ?

S.L. Je me souviens d'un accueil très positif. Bouchard a toujours été un auteur bien apprécié des gens. Maintenant, le théâtre c'est un petit milieu, c'est pas tout le monde qui va au théâtre. Ce n'est plus comme c'était, à savoir un endroit où les idées de société s'échangeaient. Michel Marc a toujours été entre le théâtre d'intérieur et les pièces d'été aux sujets plus légers. C'est sûr que le sujet de l'homosexualité, c'est quelque chose qui était peut-être plus ouvert à ce moment-là que précédemment. Mais personnellement, je n'ai jamais ressenti d'anxiété à ce sujet dans notre milieu, en théâtre. Je ne me souviens pas de toutes les critiques mais généralement, il a toujours été assez bien reçu. Ce sont de beaux textes.

M.P. Est-ce qu'au moment de cet avènement des Feluettes, les personnages féminins étaient joués par des hommes?

S.L. Non, les femmes étaient jouées par des femmes. On était dans un processus de création, on s'était regroupé entre amis pour l'aider à faire cette pièce-là. Par exemple, la duchesse était jouée par Anne-Marie Cadieux. Il me semble aussi que dans cette version-là, Bilodeau était moins présent, moins central qu'il ne l'est dans les dernières versions. Cela dit, je pense que le fait que ce soient des hommes qui jouent des personnages de femmes, dans les versions subséquentes, rajoute beaucoup à la complexité de l'idée qui est en arrière de tout ça. Parce que c'est un petit peu comme notre époque, dans le sens où l'homosexualité est admise en grande partie, mais tu as des jeunes transgenres qui prennent de plus en plus de place. En plus, ça coïncide avec les conventions théâtrales qui existaient dans les collèges québécois de l'époque : comme il n'y avait que des gars, nécessairement, les personnages de femmes étaient joués par des gars.

M.P. Qu'est-ce que tu penses, du coup, de ce qui est sorti plus tard, de cette mise en abyme de l'histoire telle que tu la connaissais, dans la prison où est enfermé Simon ?

S.L. Je trouve que c'est normal et intéressant qu'une pièce évolue. J'aime cette amélioration dans le sens de la mise en abyme, comme s'il s'agissait d'une réflexion sur l'histoire, d'un 
nouveau regard porté à cette histoire... Personnellement, j'ai un plus grand intérêt à suivre une histoire qui se déroule, qu'une histoire qui est réinterprétée. Je pense à l'adaptation du film que j'ai trouvée trop intellectualisée. Au théâtre, tu acceptes les conventions parce que ça fait partie du jeu, ça fait partie de l'intérêt. Au cinéma, les conventions théâtrales étaient à mon avis plus difficiles à intégrer dans un contexte réaliste. C'est une autre couche qui n'était pas là et qui me met un peu mal à l'aise.

\section{M.P. À quoi ressemblait Bilodeau dans les lectures ?}

S.L. Bilodeau était un personnage plutôt comique. Je pense que c'était Robert de Bellefeuille qui jouait le personnage. Comique au début voire ridicule, mais il y a bien sûr l'aspect dramatique à la fin. Dans la lecture, c'était un peu comme ça : Bilodeau est mis à l'écart, il est très dérangé par la relation entre Simon et Vallier mais aussi par rapport au fait qu'il est, lui aussi, homosexuel... Il subit ce dilemme parce qu'il n'accepte pas son homosexualité ou l'homosexualité en général. C'est un personnage qui amène une dynamique dans ce qui n'est plus qu'une histoire d'amour.

\section{M.P. Que retiens-tu de cette expérience ?}

S.L. Le défi de jouer un personnage homosexuel. Je ne suis pas homosexuel et le fait de jouer Simon n'a pas été évident. Mais c'est devenu possible quand Michel Marc nous a dit que c'était Roméo et Juliette. J'ai réussi à rentrer dans cet esprit-là, peut-être pas exactement dans le personnage de Simon tel qu'on le voit, parce que mes motivations étaient différentes, mais je m'imaginais que Vallier c'était une fille. J'avais de ces mécanismes-là parce que je pense que le désir sexuel, tu peux pas le feindre.

\section{M.P. Donc il y a une limite au pouvoir du comédien ?}

S.L. C'est pas tant une limite qu'une stratégie. Je ne suis pas le seul acteur à utiliser des motivations qui diffèrent de ce qui est décrit dans la pièce. Dans le fond, tu utilises une émotion connue pour te motiver dans ton jeu.

\section{Sur Simon :}

M.P. Dans le cadre de ma recherche, j'essaie de voir en quoi Simon peut être considéré comme un «marginal ». Comment définirais-tu le Simon que tu as connu à travers les lectures ?

S.L. Simon, je le vois comme une force de la nature, magnétique, et qui se définit en fonction du regard que les autres portent sur lui. C'est un être aimé par tout le monde (Lydia, Vallier, Bilodeau) et ce regard lui rend difficile de se définir lui-même. Tout ça fait disparaître sa personnalité à lui mais il n'est pas non plus ce que les autres voient de lui. Je trouve qu'il est un peu victime de sa nature. Je pense que les gens trop aimés ont le risque de l'être par des personnes qui leur amènent des choses négatives. Simon a aussi un petit côté justicier mais dans la lecture, il n'embrasse pas ou mord Bilodeau car c'est avant tout un idéaliste.

\section{M.P. Que pense Simon de Vallier ?}

S.L. Je pense qu'il admire sa liberté. Il admire sa culture. La comtesse et Vallier, Français immigrés, des nobles... ça c'est admirable. Mais en même temps, ils sont pauvres comme la gale. L'aspect avec lequel Simon a le plus de difficultés, c'est qu'il trouve Vallier efféminé. En fait, je suis surpris que tu portes tant d'intérêt au personnage de Simon, et pas à Vallier. Pour moi, c'est Vallier le personnage central. 


\section{Pour conclure :}

\section{M.P. Qu'as-tu retiré de cette expérience, en tant que comédien ?}

S.L. J'ai aimé le défi de jouer ce personnage complexe. Avec Michel Marc, j'ai aussi adoré qu'il ne soit pas directif. C'était peut-être parce qu'on se connaissait et que les personnages ne nous étaient pas étrangers mais on a beaucoup appris de cette expérience que j'aborde avec modestie dans le sens où je me suis vu comme un outil dans les mains de l'auteur, afin qu'il puisse sortir son personnage. Pour Les feluettes, c'était pas une création collective. Non, c'était un laboratoire où Bouchard avait à sa disposition des acteurs qui étaient payés pour incarner les scènes qui lui venaient à l'esprit. 